\title{
Heritability of model-derived parameters of beta cell secretion during intravenous and oral glucose tolerance tests: a study of twins
}

Received: 15 November 2004 / Accepted: 4 April 2005 / Published online: 24 June 2005

(C) Springer-Verlag 2005

\begin{abstract}
Aims/hypothesis: The genetic architecture of model-derived parameters of beta cell function has never been assessed. Therefore, we estimated heritability $\left(h^{2}\right)$ for model-derived phenotypes of insulin secretion in twins. Methods: Thirty-three monozygotic (MZ) and 23 dizygotic (DZ) twin pairs from the Finnish Twin Cohort Study underwent an OGTT (plasma glucose/C-peptide at 0, 30,
\end{abstract}

Electronic supplementary material Supplementary material is available for this article at http://dx.doi.org/ $10.1007 / \mathrm{s} 00125-005-1815-2$.

\section{Lehtovirta}

Department of Medicine,

Helsinki University Central Hospital,

University of Helsinki,

Helsinki, Finland

M. Lehtovirta

Folkhälsan Research Center and Research Program

for Molecular Medicine, University of Helsinki,

Helsinki, Finland

J. Kaprio

Department of Public Health, University of Helsinki,

Helsinki, Finland

L. Groop

Department of Endocrinology, Malmö University Hospital,

Malmö, Sweden

M. Trombetta - R. C. Bonadonna

Section of Endocrinology and Metabolic Diseases,

Department of Biomedical \& Surgical Sciences,

University of Verona,

Verona, Italy

M. Trombetta $\cdot$ R. C. Bonadonna

Hospital Trust of Verona (Azienda Ospedaliera di Verona),

Verona, Italy

M. Lehtovirta $(\bowtie)$

Biomedicum Helsinki,

B331a, Botnia,

P.O. Box 700, 00029 HUS Helsinki, Finland

e-mail: mikko.lehtovirta@helsinki.fi

Tel.: +358-9505171938

Fax: +358-9-47175550
$60,90$ and $120 \mathrm{~min})$. A subset of the twin pairs $(21 \mathrm{MZ} / 20$ DZ) also underwent an IVGTT (frequent sampling of plasma glucose/insulin from 0 to $60 \mathrm{~min}$ ) followed by a 160-min euglycaemic-hyperinsulinaemic clamp (45 mU · $\min ^{-1} \cdot \mathrm{m}^{-2}$ ). Mathematical modelling was applied to the IVGTT and the OGTT to assess first-phase (readily releasable insulin [RRI]) and second-phase (sigma) secretion (IVGTT), and a global index of beta cell performance (OGTT beta index). Intraclass correlation coefficients and genetic and non-genetic components for trait variances were computed to assess the $h^{2}$ of model-derived parameters. Results: The intraclass correlation coefficients in MZ twins were 0.78 for RRI, 0.67 for sigma and 0.57 for OGTT beta index. In DZ twins the correlation coefficients were $0.23,0.32$ and 0.42 , respectively. Using the most parsimonious model for each trait, the $h^{2}$ - the proportion of variance accounted for by genetic factors - was $76 \%$ (95\% CI: 53-88\%) for RRI, 28\% (34-80\%) for sigma and $53 \%(26-72 \%)$ for OGTT beta index. Conclusions/ interpretation: Our findings demonstrate that model-derived parameters of insulin secretion have a substantial genetic component and may be used in the search for genetic determinants of beta cell function in humans.

Keywords Beta cell $\cdot$ C-peptide $\cdot$ Heritability $\cdot$ Insulin Model · Twins

Abbreviations AIC: Akaike's information criteria ctct-r: cross-twin cross-trait correlation coefficient . $h^{2}$ : heritability $\cdot$ RRI: readily releasable insulin

\section{Introduction}

In genetic studies of quantitative traits it is important to measure as accurately as possible the trait of interest [1], as this affects the statistical power of the study to detect genetic effects.

In the glucose-insulin system, the gold standard for measuring insulin sensitivity is the euglycaemic-hyperinsulinaemic clamp [2], to which other measures of insulin 
sensitivity are compared [3]. Furthermore, the physiological meaning of clamp-measured insulin sensitivity, i.e. the rate of insulin-stimulated glucose utilisation, is quite akin to the effects of insulin at the cellular level, where it accelerates the rate of glucose transport and phosphorylation [4].

In contrast, much less consensus exists about the reference measures of beta cell function. First of all, the majority of studies measure insulin concentration. Insulin concentrations, however, are only a surrogate index of secretion, and actually display the net result of three different processes: (1) insulin secretion by the beta cell; (2) first-pass insulin extraction by the liver; and (3) posthepatic clearance of insulin. The latter two processes do not reflect beta cell function, display vast inter-individual variability and show marked intra-individual adjustments in response to pathophysiological changes, such as the onset or disappearance of insulin resistance [5]. Finally, to complicate the interpretation of insulin concentration data further, glucose-stimulated insulin secretion is composed of an early and a late phase [6], which are ascribed to separate molecular processes [7] and may undergo distinct, separate evolutions under several pathophysiological conditions [8-11]. These phases are physiologically at work at the same time [6], which may complicate attempts to establish the genetic and non-genetic bases of each of them.

In the last two decades, mathematical modelling of betacell-derived hormone concentration over time has progressed considerably, enabling one to safely distinguish between early and late phases of insulin secretion under experimental reference conditions, such as IVGTT [1214] and hyperglycaemic clamp [11]. Recently, we introduced a parsimonious model of C-peptide secretion during OGTT as a tool for the physiological assessment of beta cell function [15]. This model yields a compound parameter, beta index, which reflects the ability of the beta cell to increase insulin secretion in response to oral glucose and is a more stable, reproducible and discriminatory indicator of beta cell function than the classic insulinogenic index [15]. The OGTT beta index is correlated to first-phase insulin secretion $[11,15]$ and less strongly to second-phase insulin secretion [15], as conventionally assessed during IVGTT or hyperglycaemic clamp. The index can also detect alterations in beta cell function in first-degree relatives of type 2 diabetic patients with normal glucose tolerance [11] and in individuals with impaired glucose regulation or type 2 diabetes mellitus $[15,16]$.

Modelling beta cell function may provide deeper physiological insights than those provided by conventional approaches. It is, however, unknown whether any of the model-derived parameters show any degree of heritability, which would make them suitable quantitative traits for genetic analyses.

For this paper we applied mathematical modelling to a database of results of IVGTTs performed in pairs of monozygotic (MZ) and dizygotic (DZ) twins [17], to assess the heritability $\left(h^{2}\right)$ of early and late phases of glucosestimulated insulin secretion, as determined by a model of glucose-stimulated insulin secretion. We also estimated the $h^{2}$ for OGTT beta index, obtained using a database of results for OGTTs performed in MZ and DZ twins, which partially overlapped the IVGTT database. The ultimate aim of this study was to evaluate the potential usefulness of model-derived phenotypes of beta cell function in the search for quantitative trait loci of diabetes.

\section{Subjects and methods}

Subjects The Finnish Twin Cohort Study is a programme for the study of genetic and environmental determinants of chronic disease and risk factors [18]. A baseline questionnaire was administered in 1975 to twin pairs of the same sex born before 1958, with an individual response rate of $89 \%$. Twin zygosity was determined in 1975 by examining the responses of both members of each pair to two questions on the similarity of appearance at primary school age. A set of decision rules was used to classify $93.1 \%$ of the twin pairs as MZ or DZ, while $6.9 \%$ remained unclassified [19]. The questionnaire method has been shown to be highly accurate in a validation study using genetic markers [19].

Twin pairs resident in Helsinki and surrounding communities, with no diabetes according to earlier questionnaires, were randomly selected from the Twin Cohort and invited to participate in a study where OGTT (plasma glucose and serum insulin at 0 and $120 \mathrm{~min}$ ) was performed in all subjects. A subset of twin pairs also underwent either a more detailed OGTT (plasma glucose and C-peptide measured at 0, 30, 60, 90 and $120 \mathrm{~min} ; 33 \mathrm{MZ}$, 17 female/16 male; and $23 \mathrm{DZ}, 11 \mathrm{female} / 12$ male), or an IVGTT (21 MZ, six female/15 male; and $20 \mathrm{DZ}$, seven female/13 male), as described in detail in our previous work [17], or both (13 MZ, four female/nine male; and 14 DZ, five female/nine male). Data from the detailed OGTT were used for the calculation of OGTT beta index. Insulin and glucose concentrations during IVGTT were subjected to modelling according to methods explained in detail in the Appendix. The study protocol was reviewed and approved by the local ethics committee. Informed written consent was obtained from all subjects before their participation.

Study design Anthropometric measures and body composition were assessed as previously reported [17].

IVGTT and euglycaemic-hyperinsulinaemic clamp Details of the experimental procedures have been reported elsewhere [17]. Briefly, after an overnight fast, an IVGTT was performed, during which $0.3 \mathrm{~g} / \mathrm{kg}$ body weight of glucose (maximum dose $35 \mathrm{~g}$ ) was infused intravenously over 2 min, and blood samples for the analysis of plasma glucose and serum insulin were drawn at $0,2,4,6,8,10,20,30$, 40, 50 and $60 \mathrm{~min}$. Whole-body glucose uptake was quantitated by a 160 -min euglycaemic-hyperinsulinaemic (45 $\mathrm{mU} \cdot \mathrm{min}^{-1} \cdot \mathrm{m}^{-2}$ ) clamp as previously described [20]. 
OGTT After an overnight fast, each subject ingested a 75 -g glucose solution over 5 min (time $0 \mathrm{~min}$ ). Blood samples were collected for the analysis of plasma glucose, serum insulin and C-peptide concentrations at -10 and $0 \mathrm{~min}$ (baseline samples) and 30, 60, 90 and $120 \mathrm{~min}$.

Analytical methods Plasma glucose was measured in duplicate by a glucose oxidase method using a Beckman Glucose Analyzer II (Beckman Instruments, Fullerton, CA, USA). Serum insulin concentrations were measured by RIA (Pharmacia, Uppsala, Sweden) with an interassay $\mathrm{CV}$ of $5 \%$. Serum C-peptide concentrations were measured by RIA with an interassay CV of $9 \%$ (Linco, St. Charles, MO, USA). Lipids were measured as previously described [17].

Calculations Beta cell secretion during IVGTT was estimated by applying a model of glucose-induced insulin secretion to the glucose and insulin curves of each subject. This model is similar to the one proposed by us for C-peptide during hyperglycaemic clamp [11], which, in turn, was based primarily on the minimal model introduced by Toffolo et al. for C-peptide during IVGTT [13] (described in the Appendix). Both readily releasable insulin (RRI; the first peaking phase of insulin secretion during IVGTT; pmol. $\cdot^{-1}$ ) and sigma (beta cell sensitivity to glucose during the second phase of insulin secretion; $\left[\mathrm{pmol} \cdot \mathrm{l}^{-1} \cdot \mathrm{min}^{-1}\right]$ per $\left.\left[\mathrm{mmol} \cdot \mathrm{l}^{-1}\right]\right)$ were estimated by incorporating this model of insulin secretion into the commercially available software SAAM 1.1.2 (Simulation, Analysis And Modelling; SAAM Institute, Seattle, WA, USA) [21].

Beta cell secretion during OGTT was estimated by applying a model of glucose-induced insulin secretion to the glucose and C-peptide curves of each subject as previously described [15]. C-peptide kinetics are assumed to be known in each subject according to a two-compartmental model proposed by Polonsky et al. [22]. Individual parameters are calculated from population data, as shown by Van Cauter et al. [23], according to sex, age, body surface area and presence/absence of obesity or type 2 diabetes mellitus. The model of C-peptide kinetics and secretion was resolved using the SAAM 1.1.2 software, and led in all studies to unique identification of OGTT beta index (log units), a compound parameter of beta cell function during OGTT.

Numerical values of the unknown parameters were estimated using non-linear least squares. Weights were chosen optimally, i.e. equal to the inverse of the variance of the measurement errors, which were assumed to be additive uncorrelated, with zero mean and a constant $\mathrm{CV}$. In all cases the fit was good, as shown by the respective weighted residuals of insulin and C-peptide (Fig. 1). The parameters of glucose-induced beta cell secretion were estimated with good precision, as shown by the CVs of RRI $(11.7 \pm 0.9 \%)$, of sigma $(28.8 \pm 1.7 \%)$ and of OGTT beta index $(10.5 \pm 1.2 \%)$.
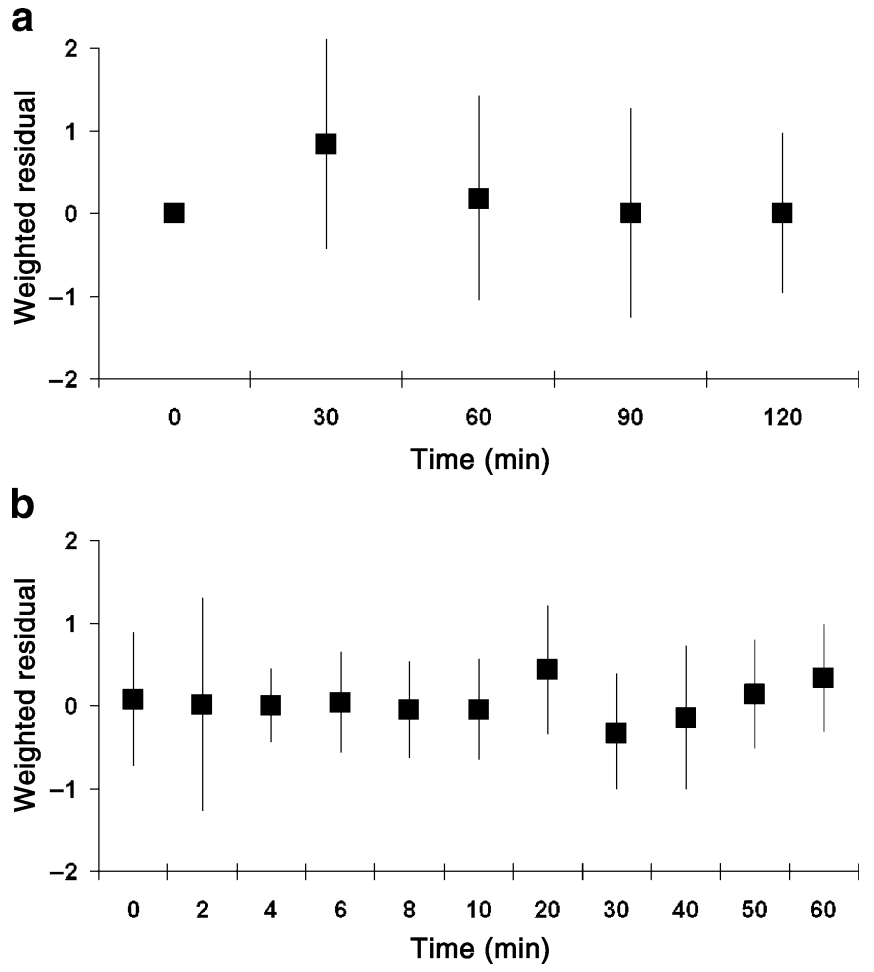

Fig. 1 Weighted residuals (means $\pm S D$, dimensionless) of model fit to plasma C-peptide (a) and insulin (b) (experimental data). The weighted residuals are the difference between measured and modelpredicted hormone concentrations divided by the standard deviation of the hormone measurement. They are a quantitative assessment of the goodness-of-fit of the models to the data. A theoretically perfect fit should generate weighted residuals with a mean 0 and an SD of 1

Intraclass correlations and model-fitting analyses To estimate genetic and environmental components of variance of the traits, standard univariate twin analyses were carried out [24]. These were based upon the principal assumptions of random mating of the twins' parents, of equal (on average) childhood environments of the $\mathrm{MZ}$ and DZ twin pairs, and of the normality of the distributions of each trait [25]. The univariate analyses included the test for homogeneity of the mean values and variances across the twin type. Maximum likelihood analyses on sample covariance matrices were used to estimate the components of variance [24]. In the current study of twins reared together, we modelled four possible components of the variance of the trait of interest, according to the maximum likelihood method: (A) an additive genetic component; (D) effects due to dominance; (C) shared environmental components; and (E) non-shared environmental components. One can fit the models based on different combinations of these parameters (AE, ACE, ADE and $\mathrm{CE}$ ). The $h^{2}$ estimates were calculated as the proportion of the variance accounted for by genetic factors (A and D) in relation to the total variance of each trait, i.e. $h^{2}=\left(V_{A}+V_{D}\right) /\left(V_{A}+V_{D^{+}}-\right.$ $\left.V_{E}\right)$ or $h^{2}=\left(V_{A}\right) /\left(V_{A}+V_{C}+V_{E}\right)$, because $V_{\mathrm{C}}$ and $V_{\mathrm{D}}$ could not be simultaneously modelled using data from twins reared together $[24,25]$. Chi square goodness-of-fit statistics 
were used to assess how well the model fitted the data. The relative goodness of fit of different models was tested using the likelihood ratio test, which is based on the fact that the more general model (in this case AE) is nested in a detailed model (for example ADE). Comparing the AE and $\mathrm{ADE}$ models gives the following likelihood ratio: the difference $\chi^{2}\left(=\chi^{2}[\mathrm{AE}]-\chi^{2}[\mathrm{ADE}]\right)$ with the difference $d f(=d f[\mathrm{AE}]-d f[\mathrm{ADE}])$. A significant likelihood ratio means that there is significant deterioration in the fit of the model when the extra parameter, D in the example, is fixed at 0 . A further comparison of the accepted models can be done by comparing their Akaike's information criteria (AIC) values, calculated as (difference $\chi^{2}-2 \cdot$ [difference $d f])[26]$.

To supplement the interpretation of the results obtained with the structural equation modelling, intraclass correlation [24] coefficients (intraclass $r$ ) for MZ and DZ pairs were compared. This is somewhat less accurate than the model-fitting procedure, because intraclass $r$ is a function of both covariance and variance; however, it lends itself to easier interpretation. In the presence of purely additive genetic variance (in addition to non-genetic, unique environment originated influences), intraclass $r(\mathrm{MZ})=2 \times$ intraclass $r$ (DZ). Shared environmental factors tend to decrease this MZ : DZ intraclass $r$ ratio, whereas nonadditive genetic factors increase it [25].

We also performed a cross-twin cross-trait correlation (ctct-r) analysis (Fig. 2) of RRI and sigma to look for evidence of any common genetic factors, as indicated by higher ctct- $r$ for $\mathrm{MZ}$ pairs than for $\mathrm{DZ}$ pairs.

Other statistical analyses Variable means are expressed as means \pm SE. The clustering of data of individuals, due to the fact that the study population consisted of pairs of twins, was taken into account in the comparison of the

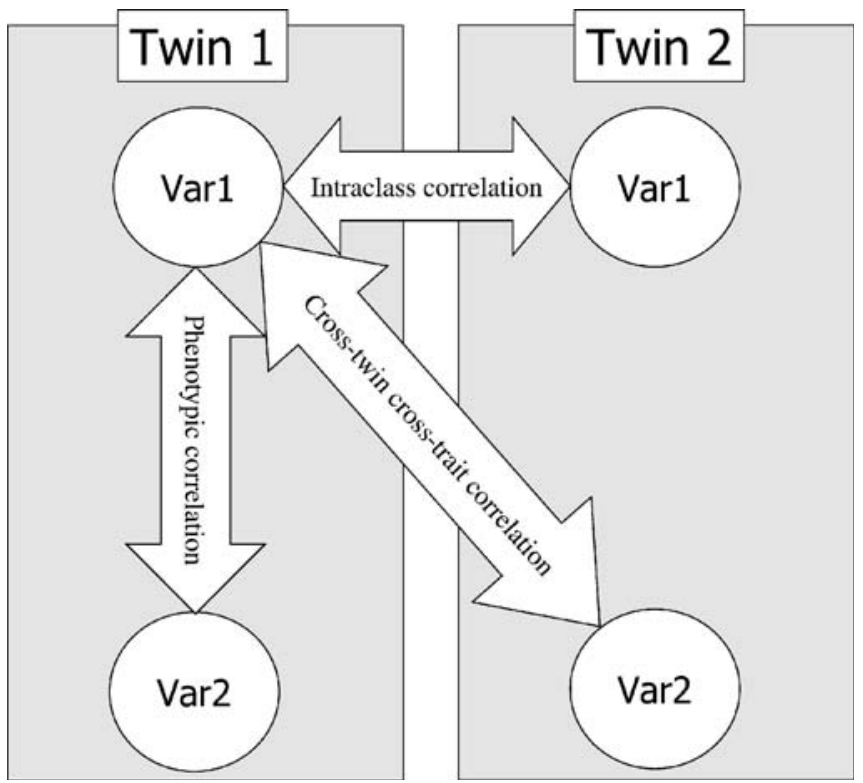

Fig. 2 A schematic representation of the relationships between phenotypic, intraclass and cross-twin cross-trait correlations. Twin 1 and twin 2 are members of a monozygotic or dizygotic twin pair mean values of the MZ and DZ twins. This was accomplished using the survey analysis properties of Stata7 statistical package (Stata Corporation, College Station, TX, USA). All trait values were adjusted for sex prior to analysis of phenotypic or intraclass correlations or of $h^{2}$. All values, except fasting glucose, OGTT beta index (already in log scale) and glucose uptake, were also log transformed to normality. The effect of the interdependence of the twin pair members on the estimation of ctct- $r$ was also controlled using the survey analysis procedures of the Stata7 statistical package. The variance component analysis, together with the estimation of $h^{2}$, was accomplished with the aid of the Mx statistical package [27]. Intraclass $r$ was calculated using TWINAN software [28]. A $p$ value was considered significant if less than 0.05 , unless stated otherwise.

\section{Results}

General characteristics of $M Z$ and DZ twins Results are shown in Table 1. There was a higher prevalence of males among the twins who underwent the IVGTT than among those who underwent the OGTT only. Dizygotic twins were older than MZ twins $(p<0.01)$, although the absolute difference was small ( $\sim 3.5$ years). In spite of similar body composition, DZ twins had higher WHR than MZ twins, showing that central adipose depots were likely to be larger in the former than in the latter. However, only glucose levels were higher in DZ than in MZ twins $(p<0.008$ $0.04)$. Both type 2 diabetes and IGT were somewhat more prevalent in DZ twins (10.3 and $31.0 \%$, respectively) than in $\mathrm{MZ}$ twins (7.3 and 18.3\%, respectively). However, clamp-measured insulin sensitivity was not lower in DZ than in MZ twins. The mean values and variances of model-derived indices of beta cell secretion were comparable in MZ and DZ twins.

Phenotypic correlations Results are shown in Table 2. RRI was inversely related to fasting and 2-h plasma glucose $(p<0.001$ for both), and directly associated with sigma $(p<0.01)$. There was no relation to insulin sensitivity. Sigma was inversely related to insulin sensitivity $(p<0.001)$, but not to glucose levels. The expected positive correlation was observed between OGTT beta index and RRI $(p<0.001)$. Furthermore, OGTT beta index was inversely related to fasting and 2-h plasma glucose $(p<0.05-0.01)$.

Intraclass and cross-twin cross-trait correlations Results are shown in Table 3. The intraclass $r$ values were significant for all traits in $\mathrm{MZ}$ twins, and for sigma in DZ twins. The coefficients were $0.57(\mathrm{MZ})$ and 0.23 (DZ) for OGTT beta index, $0.78(\mathrm{MZ})$ and $0.32(\mathrm{DZ})$ for RRI*t and $0.67(\mathrm{MZ})$ and 0.42 (DZ) for sigma. RRI*t is the readily releasable insulin during IVGTT multiplied by the time from 0 to the individual peak in insulin concentration. The square root of the value was used for the calculations.

By calculating ctct- $r$ values (Fig. 2), it is possible to investigate the existence of shared factors influencing 
Table 1 General characteristics of the $\mathrm{MZ}$ and $\mathrm{DZ}$ twins
Data are means \pm SE of the values among the $\mathrm{MZ}$ and $\mathrm{DZ}$ twins, together with the test for equality of means. The clustering of data (study population consists of twin pairs) has been taken into account in the calculation of the SEs

FFM Fat free mass, OGTT beta index beta cell ability to increase insulin secretion in response to glucose, $R R I^{*} t$ readily releasable insulin during IVGTT multiplied by the time from 0 to the individual peak in insulin concentration (square root of the value), sigma sensitivity of beta cell second-phase insulin secretion to glucose (log value)

\begin{tabular}{lccc}
\hline & MZ & DZ & $p$ value \\
\hline Number of pairs in OGTT (female/male) & $34(18 / 16)$ & $22(10 / 12)$ & \\
Number of pairs in IVGTT (female/male) & $21(6 / 15)$ & $20(7 / 13)$ & \\
Number of pairs participating in both OGTT & $13(4 / 9)$ & $14(5 / 9)$ & \\
$\quad$ and IVGTT (female/male) & & & \\
Age (years) & $63.1 \pm 4.9$ & $66.5 \pm 3.5$ & $<0.01$ \\
BMI (kg/m ${ }^{2}$ ) & $26.8 \pm 0.5$ & $26.6 \pm 0.4$ & 0.80 \\
WHR (female twins) & $0.82 \pm 0.01$ & $0.85 \pm 0.01$ & 0.04 \\
WHR (male twins) & $0.94 \pm 0.01$ & $0.98 \pm 0.01$ & 0.001 \\
Fat mass (female twins) (kg) & $22.9 \pm 1.2$ & $21.5 \pm 0.6$ & 0.30 \\
Fat mass (male twins) (kg) & $19.2 \pm 1.2$ & $19.6 \pm 1.0$ & 0.82 \\
Systolic BP (mmHg) & $140 \pm 3$ & $134 \pm 2$ & 0.09 \\
Diastolic BP (mmHg) & $83 \pm 1$ & $79 \pm 1$ & 0.04 \\
Total cholesterol (mmol/l) & $5.7 \pm 0.2$ & $5.7 \pm 0.1$ & 0.92 \\
HDL cholesterol (mmol/l) & $1.29 \pm 0.05$ & $1.41 \pm 0.07$ & 0.15 \\
Triglycerides (mmol/l) & $1.28 \pm 0.09$ & $1.42 \pm 0.12$ & 0.37 \\
Fasting plasma glucose (mmol/l) & $5.6 \pm 0.1$ & $5.9 \pm 0.1$ & 0.03 \\
Glucose at 120 min (mmol/l) & $6.8 \pm 0.2$ & $7.7 \pm 0.4$ & 0.06 \\
Fasting serum insulin (pmol/l) & $50 \pm 4$ & $48 \pm 3$ & 0.64 \\
Clamp glucose uptake $\left(\mathrm{mg} \cdot \mathrm{min}^{-1} \cdot \mathrm{kg}^{-1} \mathrm{FFM}\right)$ & $7.02 \pm 0.47$ & $8.0 \pm 0.35$ & 0.10 \\
OGTT beta index & $4.10 \pm 1.12$ & $4.33 \pm 0.16$ & 0.35 \\
IVGTT RRI (RRI*t) & $398 \pm 54$ & $301 \pm 42$ & 0.17 \\
IVGTT sigma & $3.30 \pm 0.48$ & $2.47 \pm 0.26$ & 0.20 \\
\hline
\end{tabular}

both traits. Phenotypic correlation showed a quantitatively weak $(r=0.29)$ association between RRI and sigma, suggesting that the primary determinants of these two traits are distinct. To further test this, we performed a ctct- $r$ analysis using these two traits. Despite an observed difference between the coefficients (MZ 0.28 vs DZ 0.05), neither of them reached statistical significance (Table 3 ).

Heritability estimates Results are shown in Table 4 and in the Electronic Supplementary Material [ESM]). The relative contribution of genetic factors to the variance of a quantitative trait can be expressed by $h^{2}$. It was estimated

Table 2 Phenotypic Pearson's correlation coefficients between glucose tolerance, insulin sensitivity and model-derived indices of insulin secretion

\begin{tabular}{lccccc}
\hline & FPG & G120 & M & RRI*t & Sigma \\
\hline FPG & 1 & & & & \\
G120 & $+0.48^{\mathrm{d}}$ & 1 & & & \\
M & -0.15 & -0.19 & 1 & & \\
RRI*t & $-0.37^{\mathrm{c}}$ & $-0.39^{\mathrm{c}}$ & -0.13 & 1 & \\
Sigma & -0.02 & 0.05 & $-0.38^{\mathrm{c}}$ & $+0.29^{\mathrm{b}}$ & 1 \\
OGTT beta index & $-0.27^{\mathrm{b}}$ & $-0.24^{\mathrm{a}}$ & -0.23 & $+0.45^{\mathrm{c}}$ & +0.13
\end{tabular}

FPG Fasting plasma glucose during OGTT, G120 plasma glucose during OGTT at $120 \mathrm{~min}$ (log value), $M$ clamp glucose uptake, $O G T T$ beta index beta cell ability to increase insulin secretion in response to glucose, $R R I^{*} t$ readily releasable insulin during IVGTT multiplied by the time from 0 to the individual peak in insulin concentration (square root of the value), sigma sensitivity of beta cell second-phase insulin secretion to glucose (log value)

${ }^{\mathrm{a}} p<0.05 ;{ }^{\mathrm{b}} p<0.01 ;{ }^{\mathrm{c}} p<0.001 ;{ }^{\mathrm{d}} p<0.0001$ by analysing the data according to four different possible models, as described in Subjects and methods.

Model E could be rejected for all traits (E vs other models; ESM).

For RRI, all models except $\mathrm{E}$ provided an adequate fit to the data. The model $\mathrm{AE}$ was the most parsimonious $(\mathrm{AIC}=-5.85 ; \mathrm{ESM})$. The second-best model for RRI was ADE, also including, accordingly, genetic factors due to dominance (AIC $=-3.87$; ESM). This type of model had a minimal effect on $h^{2}$, which in both cases was about $76 \%$.

For sigma, $\mathrm{AE}(p=0.035)$ and $\mathrm{CE}(p=0.036)$ models best fitted the data, but still with evidence of statistically significant deviations from the data. Although the CE model fitted the data slightly better than the AE model

Table 3 Intraclass and cross-twin cross-trait correlation coefficients in $\mathrm{MZ}$ and $\mathrm{DZ}$ twin pairs

\begin{tabular}{lcccc}
\hline Intraclass correlation & $\mathrm{MZ}$ & $p$ value & $\mathrm{DZ}$ & $p$ value \\
\hline OGTT beta index & 0.57 & $<0.0001$ & 0.23 & 0.16 \\
RRI*t $^{*}$ & 0.78 & $<0.0001$ & 0.32 & 0.08 \\
Sigma & 0.67 & $<0.0001$ & 0.42 & 0.03 \\
Cross-twin cross-trait correlation & & & \\
RRI*t-Sigma & 0.28 & $0.06^{\mathrm{a}}$ & 0.05 & $0.78^{\mathrm{a}}$ \\
\hline
\end{tabular}

OGTT beta index Beta cell ability to increase insulin secretion in response to glucose, $R R I^{*} t$ readily releasable insulin during IVGTT multiplied by the time from 0 to the individual peak in insulin concentration (square root of the value), sigma sensitivity of beta cell second-phase insulin secretion to glucose (log value)

${ }^{\mathrm{a}} p$ value from the linear regression equation between RRI and sigma in a model accounting for clustered data 
Table 4 The variance components of the best and second-best models for each trait

\begin{tabular}{|c|c|c|c|c|c|c|}
\hline & \multirow[t]{2}{*}{ Model } & \multicolumn{4}{|c|}{ Variance components } & \multirow{2}{*}{$\begin{array}{l}\text { Heritability }\left(h^{2}\right) \\
\left(V_{\mathrm{G}}\right) /\left(V_{\text {TOT }}\right)\end{array}$} \\
\hline & & $V_{\mathrm{A}}$ & $V_{\mathrm{D}}$ & $V_{\mathrm{C}}$ & $V_{\mathrm{E}}$ & \\
\hline \multirow[t]{2}{*}{ OGTT beta index } & $\mathrm{AE}$ & $53.3(25.8-72.3)$ & & & $46.7(27.8-74.2)$ & $53.3(25.8-72.3)$ \\
\hline & $\mathrm{ACE}$ & $50.0(0.0-72.2)$ & & $3.2(0.0-63.5)$ & $46.8(27.8-75.4)$ & $50.0(24.6-72.2)$ \\
\hline \multirow[t]{2}{*}{ RRI } & $\mathrm{AE}$ & $75.9(52.7-87.7)$ & & & $24.1(12.3-47.3)$ & $75.9(52.7-87.7)$ \\
\hline & $\mathrm{ADE}$ & $62.5(0.0-87.7)$ & $14.0(0.0-87.1)$ & & $24.0(12.3-47.2)$ & $76.0(52.3-87.7)$ \\
\hline \multirow[t]{3}{*}{ Sigma } & $\mathrm{ACE}$ & $27.9(0.0-78.6)$ & & $33.5(0.0-73.6)$ & $38.6(20.5-68.2)$ & $27.9(0.0-78.6)$ \\
\hline & $\mathrm{AE}$ & $62.5(34.4-79.5)$ & & & $37.5(20.5-65.6)$ & $62.5(34.4-79.5)$ \\
\hline & $\mathrm{CE}$ & & & $56.2(30.0-74.5)$ & $43.8(25.5-70.0)$ & \\
\hline
\end{tabular}

Data are percentages of overall variance $(95 \% \mathrm{CI})$. Correction for the effect of sex was applied to RRI prior to analysis

OGTT beta index Beta cell ability to increase insulin secretion in response to glucose, $R R I^{*} t$ readily releasable insulin during IVGTT multiplied by the time from 0 to the individual peak in insulin concentration (square root of the value), sigma sensitivity of beta cell second-phase insulin secretion to glucose (log value)

$V_{\mathrm{A}}$, additive variance component; $V_{\mathrm{D}}$, dominance variance component; $V_{\mathrm{C}}$, common environmental variance component; $V_{\mathrm{E}}$, unique/error variance component; $V_{\mathrm{G}}$, genetic variance of a trait; $V_{\mathrm{TOT}}$, total variance of a trait. Depending on model, $V_{G} / V_{T O T}=\left(V_{A}+V_{D} /\left(V_{A}+V_{D} V_{E}\right)\right.$ or $V_{G} / V_{T O T}=\left(V_{A}\right) /\left(V_{A}+V_{C}+V_{E}\right)$

(AIC 2.29 vs 2.38 for CE vs AE respectively; ESM), the intraclass $r$ of 0.67 in $\mathrm{MZ}$ twins $(p<0.0001)$ was in favour of the possibility that genetic factors influenced the variance of sigma. The most plausible model to explain the variance of sigma might, therefore, have been ACE if the number of twin pairs was higher. In such a case, the $h^{2}$ of sigma would have been $27.9 \%$.

For OGTT beta index, all models except E fitted the data adequately. The most parsimonious model was AE. Taking into account the MZ intraclass $r$ it seems probable that despite the AIC favouring the $\mathrm{CE}$ over the ACE model, the latter was the second-best model for OGTT beta index. The relative proportion of the component $\mathrm{C}$ was, however, no higher than 3\% when the ACE model was used to explain the variance of the trait. The two models yielded estimates of $h^{2}$ of $50-53 \%$.

\section{Discussion}

In the present study we used a twin database to assess the heritability of model-derived parameters of beta cell function obtained during an abbreviated IVGTT and a standard 2-h OGTT, as a necessary pre-requisite for future use of these quantitative traits in genetic studies. Modelling beta cell response to glucose has its strengths in dissecting out first- and second-phase insulin secretion during the IVGTT and in gauging global beta cell function during the OGTT. Nevertheless, as pointed out previously [11, 15], these are analogous models, in which parameters embed multiple physiological and molecular events underlying insulin secretion.

The characterisation of the twins participating in the IVGTT study has already been discussed in a previous paper [17]. For the analysis of OGTT beta index, twin pairs from the original set of twins studied using OGTT were included in the present paper. These were older, more obese, and had higher systolic blood pressure as well as higher fasting cholesterol, triglyceride, glucose and insulin concentrations. Accordingly, the prevalence of diabetes in the OGTT population was higher than in the IVGTT population. The removal of the twins with diabetes, however, did not change the correlations reported here (data not shown).

The phenotypic correlations of the model-derived parameters of beta cell function observed in the present report were consistent with previous studies in different populations $[11,15]$. Also, in this data set collected in Finnish twins, first-phase secretion during the IVGTT was negatively related to prevalent glucose levels, whereas secondphase secretion was inversely related to insulin sensitivity (Table 2). OGTT beta index was correlated to first-phase insulin secretion during the IVGTT and inversely related to glucose levels (Table 2). Thus, these relationships are likely to be inherent, consistent features of these model-derived parameters of beta cell function. As a corollary, the heritability figures estimated in the Finnish Twin Cohort may also be applicable to other Caucasian ethnic groups.

While intraclass $r$ suggested that RRI measured during the IVGTT was the parameter of beta cell function most influenced by apparently genetic factors, the ctct- $r$ showed little or no relationship between the model-derived parameters of beta cell function (Table 3). Since first- and secondphase insulin secretion in response to glucose are served by distinct molecular processes [7], RRI and sigma may be good quantitative traits to dissect out the specific genetic determinants of first- and second-phase insulin secretion. Importantly, the conventional assessment of first- and second-phase insulin secretion, as also done by us in a previous report [17], provides traits showing significant degrees of ctct- $r$ (data not shown), and, for this reason, are presumably less suitable for assessing the specific determinants of the two insulin secretion phases.

Intraclass $r$ also predicted a significant genetic influence on OGTT beta index (Table 3), a finding that may be secondary to the correlation with first-phase secretion. However, as this correlation accounted for about $20 \%$ of the total variance of OGTT beta index (OGTT beta index vs 
RRI; Table 2), it seems unlikely that it could have justified the whole intraclass $r$ of OGTT beta index. Furthermore, the response of the beta cell to oral glucose is under the control of many important determinants not in play during an intravenous glucose stimulus, foremost among them being the gastrointestinal incretins [29]. Finally, OGTT beta index is a global parameter, which does not discriminate between the equivalents of first-and second-phase insulin secretion [15], both of which are also conceivably elicited by oral glucose administration [30, 31].

The findings with respect to intraclass $r$ and ctct- $r$ were extended by a formal heritability analysis. The wide confidence intervals of the variance components and $h^{2}$ (Table 4) show that our study population was small. The results obtained should, therefore, not be regarded as conclusive. The most consistent finding was that RRI, the modelderived measure of first-phase insulin secretion, is controlled by genetic factors (Table 4). The figure we report here is $76 \%$, higher than the $55 \%$ found with the conventional assessment of first-phase secretion [17]. Little published data are available on insulin secretion. Lindsten et al. measured insulin concentrations from samples collected during glucose infusion in healthy twins. Their estimate of $h^{2}$ for insulin secretion was between 50 and $39 \%$, and between 17 and $44 \%$ for an interaction term of insulin and glucose [32]. The $h^{2}$ for insulin secretion (IVGTT) among women with polycystic ovary syndrome and their first-degree relatives was 74\% [33]. Recently, Poulsen et al. estimated the $h^{2}$ of the disposition index (i.e. glucose-corrected incremental insulin AUC [0-10 min] during IVGTT multiplied by insulin sensitivity obtained by euglycaemic-hyperinsulinaemic clamp) to vary between 0.75 and 0.84 in a population consisting of young and old MZ and DZ twins [34]. Due to the fact that some, but not all, of the twins in our study underwent IVGTT and clamp in a single day, it was not justified to calculate the disposition index from their data [17].

With the above-mentioned limitations in mind, we are the first to provide $h^{2}$ estimates of independent early$(R R I * t)$ and late- (sigma) phase insulin secretion obtained from a single IVGTT. Our interpretation is that modelling better separated the beta-cell-related component (i.e. firstphase secretion) from other components (insulin clearance, initial part of second-phase secretion) which may act as confounders in conventional assessments of beta cell secretion. That RRI had such a prominent genetic basis is of particular relevance, because, even when assessed conventionally, it is a strong predictor of type 2 diabetes as well as the best pathophysiological pacemaker of the deterioration in glucose homeostasis [8]. Furthermore, this finding suggests that the negative relationship between plasma glucose and model-assessed first-phase secretion [11] (Table 2) is more likely to be determined primarily by genetic determinants and/or modifiers of first-phase secretion than it is to simply mirror "glucose toxicity" on the beta cell [35]. Stated otherwise, our previous [11] and present data are more consistent with the decline in first- phase secretion being a cause of rising glycaemia than vice versa.

The same explanation used for the high $h^{2}$ of RRI holds for the apparently low $h^{2}$ of sigma, the model-derived parameter of second-phase insulin secretion, which may be as low as $28 \%$ (Table 4 ), a much less impressive figure than the one found with conventional assessment of second-phase secretion (58\%) [17]. This may also be explained by the fact that, as in previous studies [11], modelderived second-phase secretion appears to be inversely related to insulin sensitivity in a closed loop, in which second-phase secretion is the adaptive component. Since insulin sensitivity, in turn, displays a genetic component that may be as low as $37 \%$ [17], it is not surprising that second-phase secretion may have an even lower heritable component than insulin sensitivity. However, although not particularly high, the genetic component of second-phase insulin secretion is still of paramount pathophysiological importance, because it determines the appropriateness of its adaptation to changes in insulin sensitivity and, eventually, it accounts for a significant part of the inherited vulnerability of an individual to insulin resistance [36].

The finding of $h^{2}$ being as high as 53\% for OGTT beta index (Table 4) justifies future application of this index in genetic studies. The OGTT beta index is derived from a 2-h OGTT, a standard clinical tool with a limited number of samples (every $30 \mathrm{~min}$ ). It is a pure measure of beta cell function (i.e. it accounts for the glucose stimulus and is independent of insulin clearance), and has good discriminant power and reproducibility [15]. Therefore, it may also be easily applied in large-scale studies. Furthermore, OGTT can be considered the reference situation for the assessment of beta cell function, because it is the one in which clinical dysregulations of the glucose-insulin system are detected and classified [37, 38]. However, pros and cons should be carefully evaluated. Being a global index of beta cell function, OGTT beta index also collects many determinants of insulin output, i.e. first- and secondphase secretion, neural and entero-insular influences on beta cell etc. Hence, OGTT and OGTT beta index are definitely less specific than RRI and sigma.

In summary, we have reported evidence that modelderived parameters of beta cell function during IVGTT and OGTT are quantitative traits with significant degrees of heritability and with potentially richer information content than other conventional assessments of beta cell insulin secretory function [17]. Of the three parameters examined in this study, RRI, the model-derived quantitative trait describing first-phase secretion, was primarily determined by genetic influences and could be considered a phenotype suitable for genetic studies. Having acknowledged that a broader use of the approach described here requires additional research, we conclude that our findings may provide a rational basis for the use of the mathematical modelling of glucose-induced insulin secretion in the search for genetic determinants of beta cell function in humans. 
Acknowledgements This study received grants from Finska Läkaresällskapet, the Finnish Diabetes Research Society, the Research Foundation of the Orion Corporation, the Finnish-Norwegian Medical Foundation and Novo Nordisk Foundation (M. Lehtovirta); from the European Union Fifth Framework Program (QLG2-CT-200201254) under the Genomeutwin project (J. Kaprio); from the Sigrid Juselius Foundation, the Academy of Finland and the Folkhälsan Research Foundation (L. Groop); and from the University of Verona Research Grant (60\%) (R. C. Bonadonna).

The planning of the study of glucose and insulin metabolism in twins was performed in association with $\mathrm{J}$. Tuomilehto and $\mathrm{J}$. Eriksson from the National Public Health Institute, both of whom are kindly acknowledged.

The authors are indebted to S. Heikkinen, A. Tapio, M. Ben-Ami, H. Åkerfelt, T. Ilomäki, L. Toivanen and E. Laurila for their skilful assistance with the study subjects and for their analytical work. We are indebted to L. Boselli for performing the modelling analysis of the IVGTTs and the OGTTs. L. Groop has been a consultant for Bristol Myers Squibb, Aventis-Sanofi, Roche and Kowa.

\section{Appendix}

Minimal model of insulin secretion in IVGTT

The analysis of the glucose and insulin curves during the IVGTT follows the general strategy proposed by Toffolo et al. [13] with some slight changes. Since insulin kinetics cannot be computed with the experimental design employed in the present paper and, as opposed to C-peptide [22], cannot be inferred on the basis of population-derived parameters, it is assumed to be monocompartmental and all parameters of insulin secretion are normalised to the insulin distribution volume, which remains undetermined. Basal insulin secretion rate (ISRb; units: $\mathrm{pmol} \cdot \mathrm{l}^{-1} \cdot \mathrm{min}^{-1}$ ) equals basal insulin concentration multiplied by the apparent rate constant of insulin catabolism, which is calculated by the computerised analysis of the IVGTT.

IVGTT-stimulated insulin secretion, normalised to insulin distribution volume, is assumed to consist of two components: the first phase (units: $\mathrm{pmol} \cdot \cdot^{-1}$ ) is described as a square wave insulin infusion, the value of which is RRI (t) (units: $\mathrm{pmol} \cdot \mathrm{l}^{-1} \cdot \mathrm{min}^{-1}$ ), starting at time $t_{1}$ and ending at time $t_{2}$ in response to the sudden hyperglycaemic stimulus; the second phase is described by the following equations:

$$
\begin{aligned}
& \operatorname{SR}(t)=\operatorname{tau}^{-1} \cdot X(t) \\
& \begin{aligned}
d X(t) / d t & =-\operatorname{tau}^{-1} \cdot X(t)+\operatorname{sigma}[G(t)-\text { theta }] X(0) \\
& =0
\end{aligned}
\end{aligned}
$$

where $\operatorname{SR}(t)$ is the secretion rate $\left(\mathrm{pmol} \cdot \mathrm{l}^{-1} \cdot \mathrm{min}^{-1}\right)$ of insulin, tau (min) is the time constant with which the amount of insulin made available for secretion is released into the circulation, $X(t)$ is the amount of insulin made available for secretion by the glucose stimulus, sigma $\left(\left[\mathrm{pmol} \cdot \mathrm{l}^{-1} \cdot \mathrm{min}^{-1}\right]\right.$ per $\left.\left[\mathrm{mmol} \cdot 1^{-1}\right]\right)$ is the slope of the straight line relating plasma glucose $\left(\mathrm{mmol} \cdot \mathrm{l}^{-1}\right)$ to the provision of insulin $\left(\mathrm{pmol} \cdot \mathrm{l}^{-1} \cdot \mathrm{min}^{-1}\right)$ to the secretory pro- cess, $G(t)$ is the prevailing plasma glucose level (mmol/l), and theta is the plasma glucose threshold ( $\mathrm{mmol} / \mathrm{l})$ above which the second phase of insulin secretion starts. Therefore, the equations describing insulin concentration during the IVGTT are as follows:

$$
\begin{aligned}
& d I(t) / \mathrm{d} t=I \mathrm{SR} b+\mathrm{RRI}(t)+\operatorname{tau}^{-1} \cdot X(t)-k \cdot I(t) \\
& \quad \text { for } t_{1}<t<t_{2}
\end{aligned}
$$

and

$$
\begin{aligned}
& d I(t) / d t=I \mathrm{SR} b+\operatorname{tau}^{-1} \cdot X(t)-k \cdot I(t) \\
& \quad \text { for } t<t_{1} \text { or } t>t_{2}
\end{aligned}
$$

in which $I(t)$ is insulin concentration at time $t$, and $k$ is the apparent rate constant of insulin catabolism. Times $t_{1}$ and $t_{2}$ are found empirically by an iterative systematic search at discrete intervals of $0.1 \mathrm{~min}$ of the pair of values minimising the Akaike information criterion [26] of the curve-fitting process performed with SAAM II software in each study. The Akaike information criterion varies according to the following: (1) the number of adjustable parameters; (2) the total number of data points; and (3) the objective function that is minimised during the optimisation of fitting. Thus, in each study, the pair of $t_{1}$ and $t_{2}$ that minimises the Akaike information criterion is the one associated with the lowest value of the objective function, i.e. with the best model fit of the data. In three cases, minimising the AIC provided estimates of sigma with a $\mathrm{CV}$ greater than $100 \%$. In these studies, we restricted the field of acceptable solutions to those with a CV below $100 \%$ for both RRI and sigma. The solutions with the lowest AIC values were then regarded as the most parsimonious.

The parameters defining beta cell response to glucose are as follows:

- First-phase secretion, referred to in the present paper as readily releasable insulin (RRI, which equals RRI $[t] \cdot\left[t_{2}-t_{1}\right]$, units: pmol $\left.\cdot 1^{-1}\right)$

- Sigma, also named phi2 (units: $\left[\mathrm{pmol} \cdot \mathrm{l}^{-1} \cdot \mathrm{min}^{-1}\right]$ per $\left.\left[\mathrm{mmol} \cdot \mathrm{l}^{-1}\right]\right)$, which is the sensitivity of beta cell to glucose during the second phase of secretion

- Tau, the time constant (units: min) of second-phase secretion

- Theta, the glucose threshold of second-phase insulin secretion (units: $\mathrm{mmol} \cdot \mathrm{l}^{-1}$ ).

It should be noted that, since this study sampled the IVGTTs for only $60 \mathrm{~min}$, the glucose threshold was assumed to be equal to pre-test glucose concentration, and the basal insulin secretion rate was computed with pre-test insulin concentration. In a standard IVGTT analysed with minimal models, sampling is prolonged until $180-240 \mathrm{~min}$, and glucose thresholds and basal insulin secretion rates are based on the levels attained at the end of the test $[13,14]$. Thus, glucose threshold and basal insulin secretion in the minimal model analysis of the IVGTTs included in the 
present study were higher than they might have been if sampling had been continued for an additional 120-180 min.

To assess the potential impact of these differences on the RRI and sigma values, we analysed 44 IVGTTs, carried out in individuals not included in the present paper, using either all values (up to $180 \mathrm{~min}$ ) or only the samples up to $60 \mathrm{~min}$. RRI was $946 \pm 145 \mathrm{pmol} \cdot \mathrm{l}^{-1}$ with all samples and $915 \pm 133 \mathrm{pmol} \cdot \mathrm{l}^{-1}$ with samples up to $60 \mathrm{~min}(p=0.32)$; there was a very strong correlation between the two estimates $(r=0.978, p<0.001)$. The value of sigma was lower with all of the samples $\left(5.94 \pm 1.41\left[\mathrm{pmol} \cdot 1^{-1} \cdot \mathrm{min}^{-1}\right]\right.$ per $\left[\mathrm{mmol} \cdot \mathrm{l}^{-1}\right]$ ) than with just the samples up to $60 \mathrm{~min}$ $\left(7.93 \pm 1.96\left[\mathrm{pmol} \cdot 1^{-1} \cdot \mathrm{min}^{-1}\right]\right.$ per $\left.\left[\mathrm{mmol} \cdot{ }^{-1}\right] ; p<0.02\right)$ as an obvious consequence of the higher theta $(5.13 \pm 0.06$ $\left.\mathrm{mmol} \cdot \mathrm{l}^{-1}\right)$ assumed in the latter than estimated by the model in the former case $\left(4.67 \pm 0.13 \mathrm{mmol} \cdot \mathrm{l}^{-1} ; p<0.001\right)$. However, the correlation between the two estimates of sigma was also strong $(r=0.93, p<0.001)$, supporting the legitimacy of using a sigma value derived from a 60 -min IVGTT.

\section{References}

1. Flint J, Mott R (2001) Finding the molecular basis of quantitative traits: successes and pitfalls. Nat Rev Genet 2:437-445

2. DeFronzo RA, Tobin JD, Andres R (1979) Glucose clamp technique: a method for quantifying insulin secretion and insulin resistance. Am J Physiol 237:E214-E223

3. Radziuk J (2000) Insulin sensitivity and its measurements: structural commonalities among methods. J Clin Endocrinol Metab 85:4426-4433

4. Saltiel AR, Kahn CR (2001) Insulin signalling and the regulation of glucose and lipid metabolism. Nature 414:799-806

5. Mittelman SD, Van Citters GW, Kim SP et al (2000) Longitudinal compensation for fat-induced insulin resistance includes reduced insulin clearance and enhanced beta cell response. Diabetes 49:2116-2125

6. Henquin J-C, Boitard C, Efendic S, Ferrannini E, Steiner DF, Cerasi E (2002) Editorial. Insulin secretion. Movement at all levels. Diabetes 51(Suppl 1):S1-S2

7. Henquin J-C, Ishiyama N, Nenquin M, Ravier MA, Jonas J-C (2002) Signals and pools underlying biphasic insulin secretion. Diabetes 51(Suppl 1):S60-S67

8. Weyer C, Bogardus C, Mott DM, Pratley RE (1999) The natural history of insulin secretory dysfunction and insulin resistance in the pathogenesis of type 2 diabetes mellitus. J Clin Invest 104:787-794

9. Tripathy D, Carlsson AL, Lehto M, Isomaa B, Tuomi T, Groop $\mathrm{L}$ (2000) Insulin secretion and insulin sensitivity in diabetic subgroups: studies in the prediabetic and diabetic state. Diabetologia 43:1476-1483

10. Van Haeften T, Pimenta W, Mitrakou A et al (2000) Relative contributions of beta-cell function and tissue insulin sensitivity to fasting and post glucose-load glycaemia. Metabolism 49:1318-1325

11. Bonadonna RC, Stumvoll M, Fritsche A et al (2003) Altered homeostatic adaptation of first- and second-phase beta cell secretion in the offspring of patients with type 2 diabetes: studies with a minimal model to assess beta-cell function. Diabetes 52:470-480

12. Bergman RN, Phillips LS, Cobelli C (1981) Physiologic evaluation of factors controlling glucose tolerance in man: measurement of insulin sensitivity and beta cell glucose sensitivity from the response to intravenous glucose. J Clin Invest 68:1456-1467
13. Toffolo G, De Grandi F, Cobelli C (1995) Estimation of betacell sensitivity from intravenous glucose tolerance test Cpeptide data. Knowledge of kinetics avoids errors in modelling the secretion. Diabetes 44:845-854

14. Monauni T, Zenti MG, Cretti A et al (2000) Effects of glucosamine infusion on insulin secretion and insulin action in humans. Diabetes 49:926-935

15. Cretti A, Lehtovirta M, Bonora E et al (2001) Assessment of beta-cell function during the oral glucose tolerance test by a minimal model of insulin secretion. Eur J Clin Invest 31:405416

16. Del Prato S, Marchetti P, Bonadonna RC (2002) Phasic insulin release and metabolic regulation in type 2 diabetes. Diabetes (Suppl 1):S109-S116

17. Lehtovirta M, Kaprio J, Forsblom C, Eriksson J, Tuomilehto J, Groop L (2000) Insulin sensitivity and secretion in monozygotic and dizygotic twins. Diabetologia 43:285-293

18. Kaprio J, Sarna S, Koskenvuo M, Rantasalo I (1978) The Finnish Twin Registry: formation and compilation, questionnaire study, zygosity determination procedure, and research program. Prog Clin Biol Res 24:179-184

19. Sarna S, Kaprio J, Sistonen P, Koskenvuo M (1978) Diagnosis of twin zygosity by mailed questionnaire. Hum Hered 28:241254

20. Eriksson J, Franssila-Kallunki A, Ekstrand A et al (1989) Early metabolic defects in persons at increased risk for non-insulin dependent diabetes mellitus. N Engl J Med 321:337-343

21. Barrett PH, Bell BM, Cobelli C et al (1998) SAAM II: simulation, analysis, and modelling software for tracer and pharmacokinetic studies. Metabolism 47:484-492

22. Polonsky KS, Licinio-Paixao J, Given BD et al (1986) Use of biosynthetic human C-peptide in the measurement of insulin secretion rates in normal volunteers and type I diabetic patients. J Clin Invest 77:98-105

23. Van Cauter E, Mestrez F, Sturis J, Polonsky KS (1992) Estimation of insulin secretion rates from C-peptide levels. Comparison of individual and standard kinetic parameters for C-peptide clearance. Diabetes 41:368-377

24. Neale MC, Cardon LR (1992) Methodology for genetic studies of twins and families. NATO ASI Series. Kluwer Academic Publishers, Dordrecht

25. Posthuma D, Beem AL, de Geus EJC et al (2003) Theory and practice in quantitative genetics. Twin Res 6:361-376

26. Akaike H (1974) A new look at the statistical model identification. IEEE Trans Automat Contr 19:716-723

27. Neale MC, Boker SM, Xie G, Maes HH (2002) Mx: statistical modelling, 6th edn. VCU, Department of Psychiatry, Richmond, VA, USA

28. Williams CJ, Christian JC, Norton JA Jr (1992) TWINAN90: a Fortran program for conducting ANOVA-based and likelihoodbased analyses of twin data. Comput Methods Programs Biomed 38:167-176

29. Nauck MA, Bartels E, Orskov C, Ebert R, Creutzfeldt W (1993) Additive insulinotropic effects of exogenous synthetic human gastric-inhibitory polypeptide and glucagon-like peptide-1(7-36) amide infused at near-physiological insulinotropic hormone- and glucose concentrations. J Clin Endocrinol Metab 76:912-917

30. Breda E, Cavaghan MK, Toffolo G, Polonsky KS, Cobelli C (2001) Oral glucose tolerance test minimal model indexes of beta-cell function and insulin sensitivity. Diabetes 50:150-158

31. Mari A, Schmitz O, Gastaldelli A, Oestergard T, Nyholm B, Ferrannini E (2002) Meal and oral glucose tests for the assessment of beta-cell function: modelling analysis in normal subjects. Am J Physiol Endocrinol Metab 283:E1159-E1166

32. Lindsten J, Cerasi E, Luft R, Morton N, Ryman N (1976) Significance of genetic factors for the plasma insulin response to glucose in healthy subjects. Clin Genet 10:125-134 
33. Colilla S, Cox NJ, Ehrmann DA (2001) Heritability of insulin secretion and insulin action in women with polycystic ovary syndrome and their first degree relatives. J Clin Endocrinol Metab 86:2027-2031

34. Poulsen P, Levin K, Petersen I, Christensen K, Beck-Nielsen H, Vaag A (2005) Heritability of insulin secretion, peripheral and hepatic insulin action, and intracellular glucose partitioning in young and old Danish twins. Diabetes 54:275-283

35. Rossetti L, Giaccari A, DeFronzo RA (1990) Glucose toxicity. Diabetes Care 13:610-630
36. Bell GI, Polonsky KS (2001) Diabetes mellitus and genetically programmed defects in beta-cell function. Nature 414:788-791

37. The Expert Committee on the Diagnosis and Classification of Diabetes Mellitus (1997) Report of the expert committee on the diagnosis and classification of diabetes mellitus. Diabetes Care 20:1183-1197

38. WHO Consultation Group (1999) Definition, diagnosis and classification of diabetes mellitus and its complications. Report of a WHO consultation. Part 1: diagnosis and classification of diabetes mellitus. WHO/NCD/NCS/99.2, Geneva 